\title{
Dyspareunies masculines psychogènes
}

Robert GELLMAN

Psychiatre des Hôpitaux de Paris- Directeur d'Enseignement Clinique à la Faculté de Médecine PARIS VII

Claire Gellman-BarouX

Psychologue - Psychanalyste Secrétaire de l'Enseignement de Sexologie (Necker)

\section{RESUME}

La chronicisation de la douleur peut apparaître comme une énigme, puisqu'elle peut persister après un traitement somatique efficace.

En fait ce sont des traces amnésiques qui la perpétuent sous l'influence de l'appréhension et de l'angoisse qui y sont liées.

A propos des dsyspareunies masculines, nous étudierons :

- la douleur châtiment,

- la douleur appel,

- la douleur rejet de soi-même,

- la douleur dépressive,

- la douleur prétexte.

La douleur nous paraît être le point de rencontre d'une lésion organique, d'une structure de personnalité et d'un conflit qu'il soit intra-psychique ou relationnel.

Son abord devra être nécessairement pluridisciplinaire s'il se veut efficace.

Mots-clés : dyspareunie masculine, douleur châtiment, douleur appel, douleur rejet de soi-même, douleur dépressive, douleur prétexte, conflit intra-psychique, conflit relationnel.

Adresse des auteurs : E.F.S. (Ecole Française de Sexologie)

3 , rue Copernic - 75116 Paris

\section{DYSPAREUNIES MASCULINES PSYCHOGÈNES}

Classiquement la dyspareunie est la douleur survenant durant le coït chez la femme [5]. Mais ce symptôme existe également chez l'homme. Les étiologies organiques (phimosis, infections, maladie de la Peyronie...) sont exclues dans notre étude.

Comment expliquer qu'un organe destiné au plaisir puisse devenir un lieu de souffrance? Telle est la question que chaque patient consultant pour des douleurs génitales vient nous poser implicitement.

Nous allons étudier ce symptôme de douleurs génitales en fonction de différentes situations que nous sommes amenés à rencontrer en sexologie et que nous avons volontairement schématisées en cinq paragraphes :

- la douleur châtiment,

- la douleur appel,

- la douleur rejet de soi-même,

- la douleur dépressive,

- la douleur prétexte.

\section{LES DIFFÉRENTES FORMES DE DOU- LEURS VUES D'UN POINT DE VUE PSYCHOLOGIQUE}

\section{La douleur châtiment}

La douleur peut correspondre à une forme de châtiment que s'inflige le sujet et qui est liée à un sentiment de culpabilité.

Monsieur B. âgé de 48 ans, souffre par exemple, de douleurs péniennes diurnes 
depuis 14 mois, auxquelles se sont associées, peu après, des brûlures mictionnelles.

Les bilans somatiques et complémentaires se sont révélés normaux. La psychothérapie entreprise mettra en évidence l'importance de ses désirs de relations extra-conjugales. Or ces activités entraînent régulièrement une violente augmentation des douleurs. Il se censure donc lui-même grâce aux phénomènes douloureux.

\section{La douleur appel}

La douleur peut représenter une sorte d'appel au secours lorsque le sujet a ressenti, dans sa petite enfance, une carence affective. Il cherche alors à attirer l'attention de son environnement et à devenir l'objet de la sollicitude d'autrui.

M. L., par exemple, âgé de 43 ans, présente des douleurs péniennes intermittentes, à la fois diurnes et nocturnes, entraînant des réveils plusieurs fois par nuit avec érection douloureuse. Les relations sexuelles accroissent les douleurs péniennes.

Là encore, examen clinique et bilans complémentaires très minutieux n'ont permis de déceler aucune étiologie organique.

La psychothérapie entreprise montre qu'il n'existait qu'un moyen d'être pris en considération par sa mère dans l'enfance : être malade comme l'étaient ses frères et soeurs.

Ce patient présentait une libido très importante qui se heurtait à une relative frigidité de l'épouse. Ses désirs sexuels, ses masturbations et ses rêves érotiques étaient très culpabilisés. Les douleurs péniennes étaient en fait un appel à l'aide inconscient à l'égard de sa femme.

\section{La douleur rejet de soi-même}

La douleur peut stigmatiser un vécu dévalorisant de l'image corporelle. Il s'agit souvent d'hommes qui, à la suite d'accident parfois ancien, présentent de grandes déformations squelettiques ou sont défigurés à la suite d'accident de la circulation. Le rejet qu'ils ont de leur propre image et leur schéma corporel peuvent s'accompagner de dyspareunies au niveau génital.

\section{La douleur dépressive}

Elle peut correspondre à une auto-dépréciation en relation avec une perte. On peut alors retrouver ce symptôme après une perte de situation, une mise à la retraite, une faillite, en cas de transplantation le migrant exprimant par ses douleurs à la fois la perte de ses racines et ses difficultés d'insertion, dans un milieu vécu comme hostile et frustrant au point de vue sexuel.

Monsieur R., âgé de 56 ans, nous est adressé par exemple pour des douleurs périnéales et génitales importantes, le gênant dans toutes les positions et aggravées lors des érections et des relations sexuelles.

Il a été licencié de façon très injuste, selon lui, après avoir redressé, au prix d'un travail considérable l'entreprise où il avait été embauché, ceci après un premier licenciement économique.

Cette deuxième perte d'emploi va entraîner une augmentation des troubles et un épisode dépressif.

\section{La douleur prétexte}

C'est la forme la plus souvent décrite des douleurs génitales psychogènes.

Pour certains, l'angoisse liée au plaisir est telle que celui-ci ne peut en aucun cas être autorisé. La douleur, représentant un obstacle s'opposant à la libido, est un prétexte permettant d'éviter les activités sexuelles sans mettre en cause la relation affective du couple.

\section{RÉSULTATS OBTENUS DANS 45 OBSER- VATIONS PERSONNELLES RÉUNIES DURANT UNE PÉRIODE DE 5 ANNÉES.}

- Nous avons essentiellement utilisé deux techniques :

- la psychothérapie analytique dans 14 cas, - la relaxation dans 18 cas, 3 de ces patients n'ont pas donné suite à la proposition de prise en charge.

- Les critères de choix intervenants :

- l'âge du sujet, 
- ses motivations pour l'un de ces modes d'abord,

- ses possibilités de verbalisation, de mémorisation onirique, ses possibilités d'association.

- L'évolution a été appréciée au bout d'un an de traitement:

- évolution excellente avec disparition des douleurs dans 15 cas, soit $46,8 \%$ des cas. - évolution favorable avec atténuation des douleurs n'apparaissant plus qu'à certaines périodes dans 12 cas, soit $37,5 \%$ des cas. - échec dans 5 cas, soit $15,6 \%$ des cas.

\section{STRUCTURES DE PERSONNALITÉ ET DOULEURS SEXUELLES}

L'étude des diverses situations que nous pouvons rencontrer en clinique sexologique est, en fait, insuffisante et il nous paraît qu'il faille étudier les structures de personnalité que l'on peut diagnostiquer, c'est-à-dire, pour nous, la forme d'organisation que prend le Moi en fonction de l'angoisse et des modes de défense mis en place.

Nous illustrerons cette section par des observations cliniques.

\section{Structure de personnalité de type hystérique :}

caractérisée par :

- une hyperexpressivité somatique des idées, des images et des affects inconscients,

- la psychoplasticité, la suggestibilité,

- la formation imaginaire de son personnage, la mythomanie,

- les difficultés sexuelles.

Voyons par exemple l'observation de Monsieur D., âgé de 31 ans, où l'on peut parler de dyspareunie bien que la douleur ne soit pas une douleur génitale.

Motifs de la consultation :

- "je fais l'amour avec nervosité, dit-il, et ensuite, j'ai une barre à l'estomac, il me faut 15 jours pour me remettre".

De ce fait, les relations sexuelles sont très espacées : tous les 15 jours ou 3 semaines, laps de temps qui lui est nécessaire pour voir disparaître sa symptomatologie douloureuse.

Il est très angoissé, se plaint d'asthénie, d'aérocolie. Il a des douleurs précordiales et craint l'infarctus. Il aurait également un ulcère gastrique.

Il n'a pas d'érection spontanée, nous dit avoir des érections matinales et nocturnes. L'érection se maintient après l'éjaculation.

Sa vie sexuelle: Monsieur D. est marié, il s'entend bien avec sa femme et a une fille de 4 ans et demi.

Il a eu ses premières relations sexuelles à 17 ans. Il pénétrait ses partenaires sans difficulté, mais se montrait anéjaculateur. Il ne se masturbait pas jusque là, car les masturbations n'entraînaient pas d'éjaculation. En présence d'une de ses partenaires, il a éjaculé une fois spontanément. Il a voulu retrouver ce plaisir par masturbation : "ça ne venait pas, j'ai dû me crisper d'une certaine façon et j'ai pu éjaculer".

Depuis, il doit régulièrement se crisper de nouveau pour éjaculer et cela entraîne des douleurs abdominales dont il a parlé au départ.

Il a connu sa femme à 21 ans, a vécu maritalement avec elle et s'est marié à 24 ans.

Depuis 12 ans, il essaie de traiter son symptôme. Il a tenté divers traitements médicamenteux ainsi qu'une relation, des séances de yoga, une psycothérapie, sans avoir obtenu d'améliorations.

Notions biographiques : Il décrit sa mère comme une femme légère, à la limite de la prostitution. Elle aurait eu plusieurs enfants, la plupart seraient décédés en bas âge, faute de soins maternels. Lui-même et une demie-soeur auraient survécu, car ils avaient été placés dans une famille nourricière, lui de 6 mois à 14 ans.

Il n'a pas connu son père, revoit sa mère de façon très épisodique. Il dit n'avoir aucun sentiment affectif à l'égard de ses parents nourriciers. A l'âge de 14 ans, il a voulu revivre chez sa mère. Après l'essai de divers métiers et son 
mariage, il a très bien réussi comme commerçant dans une ville de province.

Etant donné l'importance de la souffrance et de l'angoisse, il accepte une psychothérapie analytique en face à face. Un certain nombre de thèmes sont rapidement abordés, concertant ses relations avec les femmes de son entourage.

Il identifie sa femme à sa mère. Par ses lapsus, il montre qu'elles sont assimilées.

D'autres thèmes sont évoqués ultérieurement :

- Une forte agressivité orale : enfant il mordait ses draps quand il pensait aux femmes.

Actuellement, il se mord sauvagement le pouce durant les rapports sexuels, fait à mettre en rapport avec une forte angoisse de castration.

- Des préoccupations concernant son image corporelle : il se trouve laid, sa mâchoire supérieure avance trop selon lui.

- Il parle beaucoup de son angoisse de mort et il assimile le plaisir à la mort.

Un souvenir est évoqué : à 12 ans et demi, c'est-à-dire au début de sa période pubertaire, il a été hospitalisé pour une appendicectomie, dans la même chambre qu'un homme de 45 ans immigré qui agonisait. Depuis il a peur de la mort.

Il n'évoque pas, à ce moment, les souhaits inconscients probables de mort envers cet homme qui aurait pu être son père, son angoisse de castration en relation avec l'intervention qu'il allait subir, ni les thèmes racistes dont il allait nous parler ultérieurement, en relation avec une liaison de sa mère.

Cet épisode de sa vie nous paraît capital pour la bonne compréhension de cette observation. Ce n'est qu'après cette séance qu'il s'autorise à parler de son père.

Lui-même porte le nom d'un homme qui a épousé sa mère ultérieurement.

Il a quitté ses parents nourriciers à 14 ans.
En septembre, au retour des vacances, il essaie de retrouver son père.

Il renonce à cette quête en octobre et veut arrêter sa psychothérapie...

Sa mère lui a dit qu'il est peut-être d'un autre homme... un homme qui s'est suicidé parce qu'il avait tué sa propre mère.

Il reviendra deux mois plus tard pour reprendre sa psychothérapie.

Quelque temps après, il aura sa première éjaculation spontanée avec sa femme, de façon détendue comme lors de la première éjaculation qu'il a connue. A cette relation sexuelle ne succédera aucun phénomène douloureux.

Nous ne pouvons nous étendre sur les détails de cette longue observation, retenons ceci :

Il s'agit d'un problème oedipien. Son épouse et sa mère sont identifiées. La femme est vue sous un double aspect. Celui de la prostituée ou celui de la vierge inaccessible.

Mais il y a chez lui un renversement des rôles traditionnellement reconnus : chez lui la mère n'est pas la sainte mais au contraire la prostituée. Cette situation est parfaitement intolérable, l'empêche de s'abandonner dans les relations amoureuses et l'oblige à maintenir sa vigilance. Celle-ci a été si importante au début de sa vie sexuelle qu'il a présenté un symptôme d'anéjaculation primaire.

Ce n'est que par des efforts musculaires intenses qu'il arrive secondairement à éjaculer, sans grand plaisir, et en satisfaisant en même temps sa culpabilité puisque l'éjaculation est suivie de douleurs pouvant persister plusieurs jours.

Ce sujet a été entièrement guéri de son symptôme par la psychothérapie.

\section{Névrose obsessionnelle}

FREUD note que le symptôme représente un mode de "défense contre les revendications libidinales du complexe d'Oedipe". (2) 
Monsieur P., un patient très obsessionnel présentait par ailleurs un symptôme d'anéjaculation, se plaignait de douleur pendant l'intromission. Lorsqu'il ressentait l'imminence de l'éjaculation le symptôme douleur était alors le prétexte d'interrompre le rapport sexuel, permettant ainsi d'échapper au danger de l'émission intra-vaginale.

On pourrait s'interroger sur les raisons pour lesquelles le Moi ne cherche pas à éviter dans la névrose obsessionnelle les différents tourments que lui inflige son Sur Moi. En fait, on retrouve dans ces cas un évitement du sentiment de culpabilité par une série d'actes expiatoires. Le Moi évite l'angoisse de castration par un châtiment atténué, par exemple la douleur. Le symptôme a parallèlement valeur de satisfaction de pulsion masochiste.

Notons par ailleurs dans cette observation une composante sadique vis-à-vis de l'épouse qui a un très grand désir de grossesse et se voit refuser cette satisfaction par l'anéjaculation de son mari.

\section{Névrose d'angoisse pure}

Elle est souvent déclenchée par un incident organique. C'est le cas de M. D... 32 ans qui, à la suite d'une rupture du frein au cours d'une relation sexuelle, avait développé une impuissance érectile et des douleurs atroces au niveau du gland, symptôme s'intégrant à un tableau typique de névrose d'angoisse.

\section{Douleurs exclusivement psychosomatiques.}

Elle prend alors la place d'un état dépressif dont elle représente l'équivalent. Elle est en rapport avec la perte de l'objet, l'autodépréciation, la culpabilité. L'angoisse au lieu d'être flottante, se fixe sur une partie du corps. C'est ce qui peut se produire après un deuil. Ici la souffrance morale habituelle est minimisée à l'extrême et déplacée sur le corps en une douleur somatique intense.
Le choix des organes génitaux n'est pas indifférent, qui entrave les relations sexuelles, établissant ainsi une liaison entre plaisir et douleur.

C'est le cas de Monsieur C... qui a de violentes douleurs du testicule gauche qui s'accompagnent d'un important œdème local. Il a d'abord consulté des urologues, qui ont pratiqué des bilans répétés (urographie I. V. examens cytobactériologiques, etc...).

Il est adressé à un premier psychiatre qui lui a prescrit des tranquillisants et des thymoanaleptiques ;

Un chirurgien lui a ensuite conseillé une intervention qu'il n'a pas acceptée.

Il consulte un gynécologue qui nous l'adresse pensant qu'il s'agit en fait d'un problème sexologique. Il nous écrit : "Monsieur C. présente essentiellement une impuissance depuis 2 ans, à laquelle sont associées des douleurs testiculaires du côté gauche avec une irradiation inguinale gauche. Ce patient est marié. Il a eu deux enfants dont le plus âgé a été tué dans un accident de voiture, il y a à peu près deux ans. Auparavant, il avait eu relativement peu de rapports. Ceux-ci se sont espacés au fil du temps et se sont arrêtés complètement. Il peut avoir des érections nocturnes et matinales.

L'examen clinique m'est apparu tout à fait normal, exception faite d'un varicocèle gauche tout à fait banal. Les divers examens paracliniques, que j'ai fait pratiquer, n'ont montré aucune anomalie.

Il a déjà consulté par ailleurs un psychiatre qui lui a donné plusieurs traitements neuroleptiques qui ont bien entendu été "très mal tolérés...".

Le patient ne nous apportera donc cette lettre que 6 mois plus tard lorsqu'il nous est envoyé par hasard par un second confrère.

Il est pied noir, a passé son enfance et sa jeunesse en Algérie où son père possédait une grosse fortune. Son père décède lorsqu'il avait 9 
ans, et, rapidement, la fortune s'effrite. L'enfant est confié à un oncle, lui même très riche, chez lequel il fait figure de parent pauvre. A 20 ans, il vient en France faire des études.

Il nous dit d'emblée que sa femme a trouvé un ami, que son ménage est fichu, qu'il a des érections matinales mais moins fortes qu'avant, que ses douleurs et ses œdèmes sont revenus depuis deux mois et qu'il n'est soulagé que par des compresses d'argile.

Il a connu sa femme à 25 ans, et n'a eu de relation sexuelle avec elle qu'après son mariage. Durant les 3 ou 4 premières années, il avait 2 ou 3 rapports par semaine : 100 dans l'année... car sa femme en tenait un décompte précis. L'épouse n'a jamais été complètement satisfaite au point de vue sexuel. Elle lui serait restée fidèle jusqu'à il y a 2 ans. Là elle aurait trouvé un autre partenaire.

On note chez Monsieur C. :

1) la dégradation progressive des relations sexuelles. L'épouse semble avoir de gros besoins, mais se montre parfaitement passive.

2) Cette situation conjugale dégradée n'a pas résisté au drame qui a bouleversé la vie du couple 2 ans auparavant c'est-à-dire la mort accidentelle du fils aîné.

C'est depuis cette époque que le patient porte cette douleur lancinante au niveau de son testicule gauche, douleur qui symboliquement traduit les stress répétés, les pertes successives qu'il a subies depuis son enfance :

- décès de son père à 9 ans,

- revers de la fortune à la suite de ce décès,

- obligation de quitter l'Algérie où il avait vécu toute son enfance,

- décès de son fils,

- séparation d'avec l'épouse.

Voyons très brièvement ce qu'il en est de la formation du symptôme et de la lutte secondaire du Moi contre le symptôme qui ont été étudiées par FrEud dans "Inhibition, Symptôme, Angoisse". [3]
Il constate par exemple que les symptômes les plus fréquents de l'hystérie de conversion paralysie motrice, contracture ou action involontaire ou encore décharge motrice, douleur, sont des processus d'investissement, soit maintenus en permanence, soit intermittents;

Le symptôme est présent dans la situation où s'est produit le refoulement et va se substituer dès lors à l'excitation perturbée en entraînant une sensation de déplaisir plus ou moins intense.

Le symptôme de douleur se produit aussi bien par l'intervention d'un élément extérieur que par association interne.

Pour lui : "Dans le cas de la douleur corporelle, il se produit un investissement élevé et qu'il faut qualifier de narcissique, de l'endroit du corps douloureux, investissement qui ne cesse d'augmenter et qui tend, pour ainsi dire, à vider le Moi". [3]

\section{CONCLUSION}

Si nous devions schématiser à l'extrême, nous reconnaîtrions 2 types de douleur :

- tout d'abord, la douleur masochiste qui est du registre pervers au sens analytique du terme. Le sujet a besoin de l'intervention de l'autre, sous forme réelle ou fantasmatique, pour déclencher une douleur de façon consciente.

- d'autre part, la douleur symptôme dépressif qui est du registre névrotique dont nous venons de voir un certain nombre d'exemples.

Cependant, les facteurs névrotiques et pervers sont intimement liés et parfois difficiles à démêler.

Notons qu'il existe une surdétermination du symptôme douloureux.

Les facteurs organiques, psychiques et relationnels sont le plus souvent étroitement liés. Il est donc nécessaire dans tous les cas de douleur, d'effectuer une enquête étiologique très complète :

- examen clinique 
- bilan complémentaire très minutieux,

- entretien centré sur la biographie du sujet et l'anamnèse de ses troubles, dans tous les cas où cela s'avère possible, un entretien avec le partenaire se révèle très riche d'enseignement.

Il ne suffit cependant pas de constater une anomalie somatique pour conclure ipso-facto qu'elle est à l'origine du symptôme douloureux.

De même l'absence de toute mise en évidence d'un facteur organique ne signe pas l'étiologie psychogène exclusive de la douleur.

La chronicisation de la douleur représente également une fréquente énigme, puisqu'elle peut persister même après un traitement somatique efficace. Cette énigme n'est peut-être qu'apparente car elle pourrait être expliquée par les traces amnésiques qui en demeurent et pourraient la perpétuer sous l'influence de l'appréhension et de l'angoisse qui y demeurent liées.

La douleur est ainsi le point de rencontre conscientisé d'une lésion organique, d'une structure de personnalité et d'un conflit qu'il soit intra-psychique ou relationnel.

Son abord devra être nécessairement pluridisciplinaire s'il se veut efficace.

\section{RÉFÉRENCES}

1. CAMBIER J. La douleur et son langage Nouv. Presse Méd. $1979,8: 3367-3369$

2. FREUD S. 3 Essais sur la Théorie de la sexualité NRF "Idées" PARIS 1962 - P. 44

3. FREUD S. Inhibition, symptôme angoisse P.U.F. PARIS 1968 - P. 101

4. Grand Dictionnaire Encyclopédique Librairie Larousse PARIS 1983 - tome 5 p. 3471

\section{SUMMARY}

Psychogenic male dyspareunia.

Robert Gellman, Claire Gellman-Baroux

Chronic pain could appear as a riddle because it still remains after an efficient physical treatment.

Anguish and memory of the pain are linked.

About men genital pain we shall study :

- pain punishment

- pain appeal

- pain reject of oneself

- depressive pain

- pretext pain

Pain seems the meeting point of an organic lesion, of a psychological building up and of a conflict.

The treatment should be multidisciplinary to be efficient.

Key words : Psychogenic male dyspareunia, pain munishment, pain appeal, pain reject of oneself, depressive pain, pretext pain. 